\title{
Morfemas derivacionais Xerente (Jê)
}

\author{
Rodrigo Guimarães Prudente Marquez Cotrim ${ }^{1}$ \\ Armando Sõpre Xerente ${ }^{2}$
}

\begin{abstract}
Resumo
Este artigo trata de processos derivacionais da língua Xerente (família Jê). Mostramos que, nessa língua, os morfemas derivacionais são de diferentes naturezas, alguns formam novos lexemas da mesma classe da base, outros formam lexemas de classes distintas. Entre os morfemas derivacionais do Xerente há os que são combináveis com (a) temas nominais, verbais e adverbiais, b) temas nominais, (c) temas verbais, e (d) temas verbais nominalizados. A presente descrição considerou estudos sobre processos derivacionais em outras línguas Jê (cf. Costa 2013; Miranda 2010, 2014; Cotrim 2016) e referenciou-se em Aikhenvald (2015) quanto a natureza funcional de processos derivacionais encontrados nas gramáticas de diferentes línguas, assim como em Rodrigues $(1953$; 1981) e em Rodrigues e Cabral (2012) sobre nominalizações em línguas Tupí.
\end{abstract}

Palavras-chave: Xerente. Macro-Jê. Morfologia. Derivação.

\begin{abstract}
This article deals with derivational processes of the Xerente language (Jê family). We show that, in this language, derivational morphemes are of different natures, some of them form new lexemes of the same base class, others form lexemes of a different base class. Among the derivational morphemes found in Xerente there are those that are combinable with (a) nominal, verbal and adverbial themes, (b) nominal themes, (c) verbal themes, and (d) nominalized verbal themes. The present description considered previous studies on derivational processes in other Jê languages (Costa 2013; Miranda 2010, 2014; Cotrim 2016) and was referenced in Aikhenvald (2015) regarding the functional nature of derivational processes found in grammars of different languages, as well as in Rodrigues (1953; 1981), and in Rodrigues and Cabral (2012) on nominalizations in Tupí languages.
\end{abstract}

Keywords: Xerente. Macro-Jê. Morphology. Derivation.

\section{Considerações iniciais}

A derivação designa, de modo geral, o processo de formação de palavras através da adição de um afixo a um tema - verbal, nominal, adverbial, por exemplo - opondo-se à composição, a qual se constitui como um processo de

\footnotetext{
${ }^{1}$ Doutor em Linguística pelo Programa de Pós-graduação em Linguística da Universidade de Brasília (PPGL-UnB). Pesquisador no Laboratório de Línguas e Literaturas Indígenas (LALLI-UnB). Professor da Universidade Estadual de Goiás - Campus Pirenópolis (UEG).

${ }^{2}$ Mestrando em Linguística pelo Programa de Pós-graduação em Linguística da Universidade de Brasília (PPGL-UnB). Pesquisador no Laboratório de Línguas e Literaturas Indígenas (LALLI-UnB).
} 
formação de palavras por meio da reunião de duas ou mais formas, e à flexão, a qual utiliza outros tipos de afixos para formar variantes da mesma palavra ou tema. Em síntese, processos derivacionais resultam na criação de novas palavras com novos significados, além de se apresentarem como opcionais (cf. Aikhenvald 2015:50). Para Aikhenvald (2015), categorias derivacionais típicas são: diminutivos e aumentativos, nominalizações de verbos e verbalização de nomes.

Processos derivacionais da língua Xerente foram tratados em Cotrim (2016), em sua tese de doutoramento. Não obstante, ampliamos aqui os dados encontrados em Contrin (Op. cit) e aprofundamos a discussão sobre o tema em colaboração com um falante nativo da língua Xerente.

\section{Propriedade típicas da derivação}

Em Aikhenvald (2015:51) são apresentadas propriedades típicas da derivação - em contraste com propriedades flexionais, dentre as quais se destacam: (a) possui ocorrência opcional; (b) caracteriza-se por um processo pré-final - se afixo, tende a ocorrer entre a raiz e a flexão; (c) deriva um tema sujeito a flexões; (d) geralmente é específica a uma classe de palavras; (e) deriva um tema de uma classe de palavra diferente, ou acrescenta alguma especificação semântica a uma raiz, sem mudança de classe; (f) nunca indica a relação gramatical entre palavras ou participa na concordância; (g) frequentemente apresenta lacuna no paradigma; (h) amiúde semanticamente irregular; (h) pode formar sistemas amplos; (i) propensos a ter uma frequência menor; e (j) pode ser monossilábico ou mais extenso.

Um novo nome pode ser derivado a partir de um membro de uma outra classe de palavra. Processos derivacionais incluem a formação de nomes a partir de verbos resultando em deverbais que podem descrever uma atividade ou o seu resultado. Além disso, um nome pode ser derivado a partir de outro nome. Aqui incluem-se, processos derivacioanis que contribuem com noções de diminutivos - referindo-se a pequenos objetos e pessoas - e aumentativos - em sentido oposto aos diminutivos (Aikhenvald 2015: 122).

Ao contrário da terminologia utilizada por Aikhenvald (2015), preferimos fazer uso dos termos 'atenuativo' e 'intensificador', tendo em vista que os termos 'diminutivo' e 'aumentativo' não conseguem incluir especificações semânticas da língua em questão que vão além de se referirem a objetos ou a pessoas com extensões grandes ou diminutas.

\section{Processos derivacionais em Xerente}

São 11 os morfemas derivacionais do Xerente, os quais contribuem para a derivação de novos lexemas na língua, afixando-se a temas nominais, verbais e adverbiais. Esses morfemas são de diferentes naturezas e possuem funções 
específicas na língua, conforme sintetizados no Quadro 1 abaixo, extraído de Cotrim $(2016)^{3}$.

\section{Quadro 1: Morfemas derivacionais Xerente}

\begin{tabular}{|c|c|c|c|c|}
\hline $\begin{array}{c}\text { Morfema } \\
\text { derivacional }\end{array}$ & Tipo & Função & Exemplo & Tradução \\
\hline -re & Atenuativo & $\begin{array}{l}\text { Tamanho; quantidade; } \\
\text { afetividade; fases } \\
\text { de idade; tempo } \\
\text { cronológico, tipos de } \\
\text { animais (peixes, aves e } \\
\text { mamíferos) }\end{array}$ & waw $\tilde{\varepsilon}-r e$ & 'velhinho' \\
\hline -zawre & $\begin{array}{l}\text { Intensifica- } \\
\text { dor }\end{array}$ & Intensificador & $z a w r \varepsilon-\mathrm{di}$ & $\begin{array}{l}\text { 'ser/estar } \\
\text { grande' }\end{array}$ \\
\hline -kta $\sim$-ktab & $\begin{array}{l}\text { Intensifica- } \\
\text { dor }\end{array}$ & $\begin{array}{l}\text { Intensificador de } \\
\text { referentes de nomes } \\
\text { de qualidade e de } \\
\text { sensações }\end{array}$ & $k t a b i$-di & 'demasiado' \\
\hline -hu & $\begin{array}{l}\text { Nominal- } \\
\text { izador }\end{array}$ & $\begin{array}{c}\text { Nominalizador } \\
\text { existencial de tipos de } \\
\text { animais }\end{array}$ & ktã-hu & $\begin{array}{l}\text { 'manada de } \\
\text { anta' }\end{array}$ \\
\hline -nõpro & $\begin{array}{l}\text { Nominal- } \\
\text { izador }\end{array}$ & $\begin{array}{c}\text { Nominalizador } \\
\text { existencial de tipos de } \\
\text { plantas }\end{array}$ & $\begin{array}{l}\text { wde+kru+k- } \\
\text { rãĩ-hu nõprə }\end{array}$ & 'melancial' \\
\hline -nõpro-si & $\begin{array}{l}\text { Nominal- } \\
\text { izador }\end{array}$ & $\begin{array}{c}\text { Nominalizador } \\
\text { existencial de tipos de } \\
\text { plantas }\end{array}$ & $\begin{array}{l}\text { kupa+wde- } \\
\text {-hu nópro-si }\end{array}$ & 'mandiocal' \\
\hline $\begin{array}{l}-\mathbf{r i} \sim-\mathbf{r i} \sim-\mathbf{r} \\
\sim-\varnothing\end{array}$ & $\begin{array}{l}\text { Nominal- } \\
\text { izador }\end{array}$ & $\begin{array}{c}\text { Nominalizador de } \\
\text { nomes ação }\end{array}$ & kmẽ-ri & '(o) catar' \\
\hline$-k w a$ & $\begin{array}{l}\text { Nominal- } \\
\text { izador }\end{array}$ & $\begin{array}{l}\text { Nominalizador de } \\
\text { nomes de agente }\end{array}$ & $\begin{array}{c}\mathrm{da}=\text { kahu-r- } \\
\text {-kwa }\end{array}$ & $\begin{array}{l}\text { 'comedor de } \\
\text { gente' }\end{array}$ \\
\hline$-\mathbf{z \varepsilon}$ & $\begin{array}{l}\text { Nominal- } \\
\text { izador }\end{array}$ & $\begin{array}{c}\text { Nominalizador de } \\
\text { nomes de circunstância }\end{array}$ & $\begin{array}{c}\mathrm{da}=\text { nmrã- } \varnothing- \\
-\mathrm{z} \varepsilon\end{array}$ & $\begin{array}{l}\text { 'lugar de assen- } \\
\text { to (de gente)' }\end{array}$ \\
\hline$-\mathbf{d i} \sim-\mathbf{t i} \sim-\mathbf{k i}$ & $\begin{array}{l}\text { Nominal- } \\
\text { izador }\end{array}$ & $\begin{array}{c}\text { Nominalizador de } \\
\text { predicados existenciais }\end{array}$ & sdakro-ki & $\begin{array}{l}\text { '(fazer) sol } \\
\text { quente' }\end{array}$ \\
\hline$-k \tilde{0}$ & Privativo & Privativo & stikrui-kõ-di & $\begin{array}{l}\text { ‘tranquilo’ (lit.: } \\
\text { “sem raiva”) }\end{array}$ \\
\hline
\end{tabular}

3 As abreviaturas utilizadas neste estudo são as seguintes: 3 'terceira pessoa'; ASS 'associativo'; ATN 'atenuativo'; CIRC 'circunstância (nominalizador de)'; DAT 'dativo'; DES 'desiderativo'; EST 'estativo'; GEN 'genérico'; GEN.HUM 'genérico humano'; HORIZ 'horizontal'; INTENS 'intensificador'; NAG 'nominalizador de agente'; NE 'nominalizador existencial'; NNA 'nominalizador de nome de ação'; OBJ 'objeto'; PERT 'pertence'; POSS 'posse'; PRIV 'privativo'; REFL 'reflexivo'; + 'fronteira de palavras (compostos)'. 


\section{i. Atenuativo $\{-$ re $\}$}

\section{Atenuativo combinado com temas nominais}

$\mathrm{O}$ atenuativo $\{$-re $\}$ aparece em temas nominais, temas verbais nominalizados e em temas adverbiais. $\mathrm{O}$ atenuativo agregado a temas nominais expressa noções de tamanho (diminuto) de coisas e seres no mundo, mas atenua também noções de quantidade, afetividade e fases de idade das pessoas. É usado na formação de nomes de tipos específicos de animais, como aves e peixes, e de plantas presentes no universo Xerente, bem como pode contribuir na formação de nomes de partes do dia e da noite.

\section{Tamanho}

(01) a. kri-re

casa-ATN

'casinha'

b. wde-re

pau-ATN

'árvorezinha' (pequena/ jovem)

c. waptə-re

esteira-ATN

'esteirinha' (esteira pequena)

d. wabu-re

talo.de.buriti-ATN

'talinho'

e. sru-re

pequeno-ATN

'pequenininho; pouquinho; novinho (em fase de crescimento) $)^{4}$

(02) a. siw

ave

'ave (genérica)'

b. si-re

ave-ATN

'passarinho'

\footnotetext{
${ }^{4}$ Indica tamanho, quantidade ou idade.
} 

c. si-re-re
ave-ATN-ATN
'passarinhozinho'
d. si-re $\varnothing \quad$ kra-re
ave-ATN POSS cria-ATN
'filhote de passarinho'
e. si-re-re $\varnothing \quad$ kre-re
ave-ATN-ATN pOSS ovo-ATN
'ovinho de passarinhozinho'
(03) a. hi-re
fino-ATN
'fininho'
b. wapu-re
leve-ATN
'levinho' (muito leve)
c. po-re
largo-ATN
'largozinha' (largura pequena)
d. b’bate-re
estreito-ATN
'estreitinho' (1, espaço entre coisas)
e. ware-re-di
estreito-ATN-EST
'estreitinho' (para local e objetos com largura estreita como árvore, peixe, etc.)

(04) a. srã-re

montanha-ATN

'monte, montinho'

b. bru-re

roça-ATN

'rocinha (roça pequena)'

c. tkai-re

terra-ATN

'terrinha' (terreno de tamanho pequeno; lote) 
(05) a. spokre-re orelha-ATN 'orelha pequena'

b. da+pra-re HUM+pé-ATN 'pezinho' (pé pequeno de gente)

\section{Afetividade}

(06) a. tare-re criança-ATN 'menininha'

b. ture-re criança-ATN 'menininho'

(07) a. mãra wẽ-re noite bonito-ATN 'noite bonitinha'

b. wakti-re preto-ATN 'pretinho' (pessoa ou animal)

c. kwa pre-re fronte vermelho-ATN 'queimadinho de sol' (lit.: “cara vermelhinha”)

\section{Idade}

(08) a. aikde criança 'criança' (criança adolescente, “jovem”)

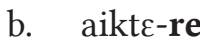
criança-ATN 'criancinha' (neném; começando andar) 
(09) a. aikte pre

criança vermelho

'recém-nascido'

b. aikte pre-re

criança vermelho-ATN

'bebezinho' (criança de colo)

(10) a. wapt $\varepsilon$

jovem

'jovem, adolescente, adulto jovem’

b. waptem-re

jovem-ATN

'jovenzinho (pré-adolescente)'

(11) a. $\quad \tilde{1}-\mathrm{pto}-\mathrm{k}(\mathrm{r}) \mathrm{da}$

3-broto-antigo

'adulto maior; senhoril ( \pm 50 anos)'

b. $\tilde{\mathrm{i}}$-pto-k(r)ta-re

3-broto-antigo-ATN

'adulto jovem (de 30-45)'

(12) a. waw $\tilde{\varepsilon}$

velho

'velho'

b. waw $\tilde{\varepsilon}-\mathbf{r e}$

velho-ATN

‘velhinho' (velho senil, velhinho)

O último exemplo (12b) se destaca porque, ao contrário dos outros em que o atenuativo -re expressa uma idade menor, "mais jovem", o uso do atenuativo nesse exemplo possui a função de expressar uma idade mais avançada, senil.

\section{Espécies animais}

(13) a. tpe ka-re

peixe branco-ATN

'piaba' (lit.: "peixe branquinho") 

b. tpe $\varnothing \quad$ kra-re peixe poss cria-ATN 'filhote de peixe'

(14) a. wapsã

cachorro

'cachorro'

b. wapsã-re

cachorro-ATN

'cachorrinho' (raça pequena)

c. wapsã $\varnothing \quad$ kra-re

cachorro POSS cria-ATN

'filhote de cachorro'

(15) a. sika

galinha

'galinha'

b. sika-re

galinha-ATN

'galinha-da-Índia' (pequena)

c. sika $\varnothing \quad$ kra-re

galinha POss cria-ATN

'pintinho (lit.: "filhote de galinha")'

d. sika krere-re

galinha macho-ATN

'garnizé, galo-anão (de raça pequena)

(16) a. ponkẽ-res

veado-ATN

'veado-galheiro' (espécie de veado)

b. sipsim-re

tatu-ATN

'tatu-sino' (espécie de tatu)

c. kuti-re

sapo-ATN

‘sapinho' (espécie de sapo)

${ }^{5}$ Em alguns contextos como neste, o sufixo atenuativo $\{$-re $\}$ pode designar, também, o “animal fêmea”. Designa, portanto, no processo derivacional, o sexo do animal (cf. Cotrim 2016). 


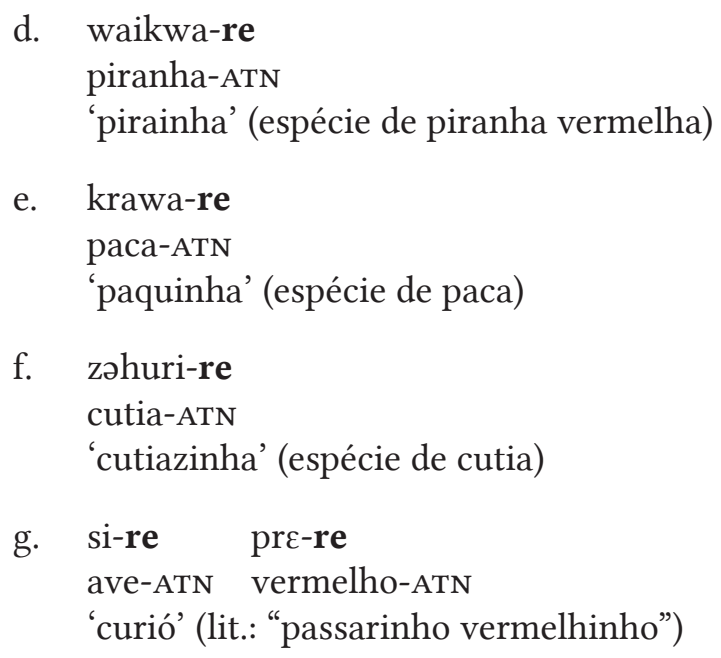

\section{Partes do dia/noite}

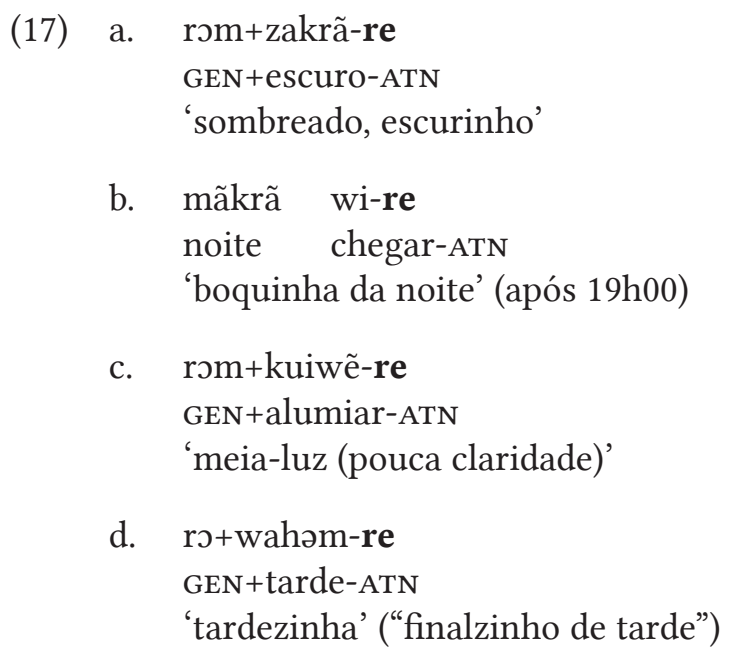

\section{Atenuativo combinado com temas verbais nominalizados}

(18) a. wakẽ-Ø-re caçoar-NNA-ATN

'(o) caçoarzinho'

b. wapska-Ø-re derrubar-NNA-ATN

‘(o) derrubarzinho' (de brincadeira) 
c. waptkã-Ø-re

cair-NNA-ATN

‘(o) cairzinho’ (cair leve algo/alguém)

d. wakre-Ø-re

furar-NNA-ATN

‘(o) furarzinho' (pouquinho)

e. wasku-Ø-re

contar-NNA-ATN

‘(o) contarzinho' (contar um conto, uma historieta de forma afetiva)

(19) a. zekrnẽ-Ø-re

beber-NNA-ATN

'beberzinho' (bebericar pouquinho)

b. nõk(r)ko-Ø-re

vomitar-NNA-ATN

'vomitarzinho' (pouquinho)

c. spokrwa-Ø-re

aviso-NNA-ATN

‘avisarzinho’ (aviso rápido, breve)

d. wra+t $t^{2} t e-\varnothing$-re

correr+firme-NML-ATN

'correrzinho' ((o) correr bem)

e. ti-br ${ }^{\circ} b a-\varnothing$-re

3-dançar-NNA-ATN

'dançadorzinho' (o que sabe dançar bonitinho), expressando

afetividade

(20) a. kmẽsi- Ø-re

comer-NNA-ATN

'comerzinho' (comer pouquinho)

b. knẽ-Ø-re

engolir-NNA-ATN

'engolirzinho' (pouquinho)

c. $\quad n^{\supset} m r a ̃-\varnothing-r e$

sentar-NNA-ATN

'sentarzinho' (afetividade) 


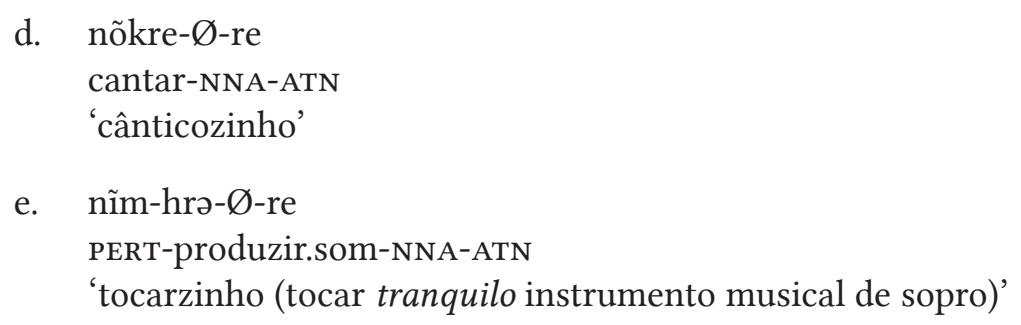

(21) a. n(õ)tõ-Ø-re

dormir-NNA-ATN

'dormirzinho' (afetividade)

b. dartõ- $\varnothing$-re

pular-NNA-ATN

'saltarzinho' (curto espaço)

c. nĩwai- $\varnothing$-re

pedir-NNA-ATN

'pedirzinho' (afetividade)

d. kwa+kre-Ø-re

afiado+buraco-NNA-ATN

'ferirzinho' (de leve)

e. nĩm-kras-Ø-re

PERT-fazer.barulho-NNA-ATN

‘fazer barulhinho' (de instrumento musical como teclado, maracá

e violão)

(22) a. duptoi-Ø-re

inflar-NNA-ATN

'inflarzinho' (encher pouco)

b. wankõ- $\varnothing$-re

atirar-NML-ATN

'atirarzinho' (atirar uma coisa pequena)

c. wawi-Ø-re

riscar-NNA-ATN

'riscarzinho'(riscar pequeno)

d. stikrui- Ø-re

zangar-NNA-ATN

'zangarzinho' (pequena raiva, aborrecimento) 
e. simãzai- $\varnothing$-re

mentir-NNA-ATN

'mentirzinho' ("mentirinha boba": grau de veracidade)

(23) a. kuikre-Ø-re

escrever-NNA-ATN

'escreverzinho' (escrita/texto pequeno)

b. srẽ-Ø-re

colocar-NNA-ATN

'colocarzinho' ( algo pequeno em um lugar)

c. katka-Ø-re

bater-NNA-ATN

'trautearzinho' (bater repetido de leve)

d. kazəi-Ø-re

bater-NNA-ATN

'baterzinho' (bater pouco, bater de leve)

e. $\mathrm{da}=$ mrmẽ- $\varnothing$-re

HUM=falar-NNA-ATN

'falarzinho' ("falar coisas pequenas, tolas, sem importâncias; fuxicar")

(24) a. pke- Ø-re

estapear-NNA-ATN

'estapearzinho' (estapear suave)

b. k-mã=wamnãi- $\varnothing$-re

3-DAT=tatuar-NNA-ATN

'tatuarzinho'

c. wsi-Ø-re

chegar-NNA-ATN

'chegarzinho' (chegar devagar)

d. $\quad k a=h(i)-r i-r e$

$3=$ cozinhar-NNA-ATN

'cozinharzinho'

e. $k a=w a k r \jmath-\varnothing-r e$

3-aquecer-NNA-ATN

'esquentarzinho' (esquentar pouco) 
(25) a. wahə-Ø-re

esfriar-NNA -ATN

'esfriarzinho' (esfriar pouco)

b. wakui- $\varnothing$-re

escaldar-NNA-ATN

'escaldarzinho' (escaldar leve)

c. $\quad$ w(ĩ)-rĩ-re

matar-NNA -ATN

'matarzinho' (matar pouco/caça pequena)

d. sdãm-Ø-re

fechar-NNA-ATN

'fecharzinho' (algo pequeno)

e. waikrãm-Ø-re

encontrar-NNA-ATN

'encontrozinho' (encontro carinhoso)

f. waikrãm- $\emptyset=$ sku-re

encontrar-NNA=rápido-ATN

'encontrozinho' (rapidinho)

\section{Atenuativo combinado com temas adverbiais}

Apresentamos, a seguir, exemplos de temas adverbiais combinados com o atenuativo $\{$-re $\}$ :

(26) a. ktãm-re

próximo-ATN

'pertinho'

b. azanã-re

depressa-ATN

'depressinha'

c. tokto-re

agora-ATN

'agorinha'

(27) a. durc-re

novamente-ATN

'de novozinho' 
b. awasi-re

depois-ATN

'depoiszinho'

Apresentamos, nesta seção, o atenuativo \{-re\} combinado com temas nominais, temas verbais nominalizados e com temas adverbiais. A sua função básica na língua é a de expressar coisas pequenas, diminutas e atenuadas no mundo. Não obstante, este morfema derivacional é responsável, ainda, por expressar afetividade e contribuir na formação de palavras que expressam quantidade, categorização de espécies animais, estágios de idade e partes do dia e da noite.

Na próxima seção apresentamos o morfema derivacional em oposição a este: o morfema intensificador $\{$-zawre\}.

\section{Morfema intensificador $\{$ zawre\}}

O morfema intensificador do Xerente $\{$-zawre\}, ao acompanhar nomes de qualidade, intensifica a característica atribuída a determinado elemento, como "(ser) grande, grosso, comprido". Por outro lado, ao se sufixar a nomes comuns, seguido do morfema estativo \{-di\}, contribui com um sentido intensificado, de quantidade e / ou de coletividade:

(28) a. painõ pa $\quad$-zawre $\quad$-di

braço comprido INTENS EST

'braço grande comprido'

b. da hi pa -zawre -di

GEN.HUM osso comprido INTENS EST

'perna comprida de gente'

(29) a. akwẽ -zawre -di

gente INTENS EST

'multidão'

b. si-re -zawre -di

ave-ATN INTENS EST

'bando de passarinho'

c. kuhə -zawre -di

porcão INTENS EST

'manada de porcão' 
(30) a. zəhuri -zawre -di

cutia INTENS EST

'muita cutia (quantidade)'

b. krawa -zawre -di

paca INTENS EST

'muita paca (quantidade)'

c. ktə -zawre -di

anta INTENS EST

'muita anta (quantidade)'

d. patre -zawre -di

mabira INTENS EST

'muita mabira (quantidade)'

(31) a. kumdi -zawre -di

batata INTENS EST

'muita batata (quantidade)'

b. mõkõnĩ -zawre -di

inhame INTENS EST

'muito inhame (quantidade)'

c. kupa -zawre -di

mandioca INTENS EST

'muita mandioca (quantidade)'

d. karos -zawre -di

arroz INTENS EST

'muito arroz (quantidade)'

\section{Morfema intensificador de referentes de nomes de qualidade e de sensações $\{-k t a \sim-k t a b\}$}

O segundo tipo de morfema intensificador do Xerente se agrega a nomes de qualidade e de sensações para expressar um grau de intensidade. Há, ainda, outro tipo de uso deste morfema intensificador, o qual é sufixado a nomes comuns, caso em que a expressão contém o morfema privativo $\{$-kõ $\}$ e o estativo $\{-$ di $\}$ resultando em algo "não muito verdadeiro ou puro".
a. k-mã+dkə pse
-kta $\sim$-ktab -di
3-DAT+olhar bom, bonito
INTENS
EST
'tratar bem' (lit.: "olhar muito bom/bonito a alguém") 

b. rom+zakrã -kta $\sim$-ktab -di
GEN+escuro INTENS EST
'muito escuro'

(33) a. te wẽ -kta $\sim$-ktab -di

3.OBJ bonito INTENS -EST

'gostar muito (de algo); achar bonito (algo)'
b. ro wẽ -kta -ktab -di
GEN bonito INTENS EST
'lugar (bem) bonito'

Nos exemplos, a seguir, o intensificador $\{-\mathrm{ktab}\}$ é posposto a nomes comuns (de aves e peixes, por exemplo), bem como a nomes que se referem a termos de parentesco e, precedendo uma "negação estativa", formada a partir do privativo $\{-k \tilde{o}\}$ e do estativo $\{$-di $\}$, expressa o sentido de que algo não verdadeiro ou genuíno:

$\begin{array}{lllll}\text { (34) a. } & \text { tpe } & -\mathrm{ktab} & -\mathrm{k} \tilde{} & -\mathrm{di} \\ & \text { peixe } & \text { INTENS } & \text { PRIV } & \text { EST }\end{array}$

'não é peixe verdadeiro'

b. wai -ktab -kõ -di

papagaio INTENS -PRIV EST

'não é papagaio verdadeiro'

c. akwẽ -ktab -kõ -di

gente INTENS PRIV EST

'não-indígena adotivo'

(35) a. $\tilde{1}-\mathrm{kra} \quad-\mathrm{ktab} \quad$-kõ $\quad$-di

3-filho INTENS PRIV EST

'filho adotivo (de alguém)'

c. amke -ktab -kõ -di

cobra INTENS PRIV EST

'cobra falsa'

d. in$_{\text {-pnãi }} \quad-k t a b \quad-k o ̃ \quad-d i$

3-irmão INTENS PRIV EST

'irmão mais novo adotivo'

e. $\tilde{\mathbf{1}}$-nĩ $\quad-k t a b \quad-k \tilde{a} \quad-d i$

3-carne INTENS PRIV EST

'carne (de algo) que não é pura; carne (de algo) com osso' 


\section{Nominalizador existencial $\{-h u\}$}

O nominalizador existencial $\{-\mathrm{hu}\}$ se combina com nomes de animais e de plantas a fim de formar predicados existenciais que expressam algo que "existe em abundância", em coletivo:

\section{Animais}

(36) a. zəmhu+pre-hu

formiga+vermelho-NE

'formigueiro'

b. mãsa-i-hu

formigão-?-NE

'formigueiro de formigão'

c. zəmhu+krã-hu

formiga+cabeça-NE

'formigueiro de formiga preta'

(37) a. ktã-hu

anta-NE

'manada de anta'

b. kaktõ-hu

pomba-NE

'bando de pomba'

c. wrã $+k u-h u$

tatu+fino-NE

'manada de tatu'

d. krawa-hu

paca-NE

'manada de pacas'

\section{Plantas}

(38) a. wakro-hu

pati-NE

'patizal'

b. krito-hu

mangaba-NE

'mangabl' 

c. kbare-hu
pequi-NE
'pequizal'
d. moktərã-hu
caju-NE
'cajuzal'

(39) a. rbekras-hu

miridiba-NE

'miridibazal'
b. nrõ+wde-hu
côco+pau-NE
'coqueiral'
c. wde+krã-i+po-hu
pau-fruto-?-chata-NE
'mangabal'

d. pizu+wde-hu

buriti+pau-NE

'buritizal'

\section{Nominalizador existencial \{nõpro\}}

Outro nominalizador existencial encontrado em Xerente é \{nõpro\} e \{nõpro-si\}, os quais se agregam, também, a nomes de plantas e de animais. De acordo com Cotrim (2016:112) "um tema derivado por meio do existencial \{-hu\} pode ser modificado pelo nome \{nõpro\}, que contribui com o significado de "horizontal", quando a planta se distribui horizontalmente pelo chão. Alguns exemplos são:
(40) a. wde+kru+krãĩ-hu nõpro
pau+rama+fruto-Ne horiz 'melancial'
b. kupa+wde-hu nõpro-si mandioca+pau-NE horiz-em.pé 'mandiocal'
c. nãnmã-hu nõpro-si
milho-NE horiz-em.pé 'milharal'


Conforme Cotrim (2016:112), nos dois últimos exemplos acrescenta-se o sufixo $\{$-si $\}$ ao morfema existencial horizontal, "para distinguir o que é visto como horizontal, mas com hastes ou "em pé", típicas das plantas rasteiras e baixas".

Além dos exemplos dados anteriormente encontramos, ainda, a combinação \{nõpro-si\} acompanhando nomes que não são termos para plantas e que não acompanham o nominalizador existencial $\{-\mathrm{hu}\}$, conforme demonstrado nos exemplos que seguem.
a. sika $\varnothing$ nĩ nõpro-si 'carne pura de frango' galinha poss carne horiz-em.pé
b. kuhəbə $\varnothing$ nĩ nõpro-si 'carne de porco puro' porco poss carne horiz-em.pé
c. ktəkmõ $\varnothing$ nĩ nõpro-si gado poss carne horiz-em.pé 'carne de gado puro'
e. krawa $\varnothing$ nĩ nõpro-si paca poss carne horiz-em.pé 'carne de paca pura'

\section{Sufixos nominalizadores}

Em Xerente, assim como em outras língua Jê, nominalizações são muito produtivas e podem ser observadas como uma das características mais proeminentes da morfologia (cf. Costa 2013; Miranda 2010, 2014; Cotrim 2016). Há três processos derivacionais que derivam temas nominais a partir de verbos, conforme demonstramos, a seguir.

\section{Nomes de ação}

De acordo com Cotrim (2016:113), a nominalização que resulta em 'nome de ação' tem como base verbos intransitivos e transitivos combinados com o morfema 'nominalizador de nome de ação' $\{$-ri\}, cujos alomorfes são: $\{-$-rĩ -ri $\sim-\mathrm{r} \sim-\varnothing\}$

Os nomes que resultam deste processo são base para outras nominalizações na língua Xerente. Exemplos de nomes de ação são dados a seguir.

$\{-$ rĩ $\sim$-ri, $\sim-r$ - Ø\} 'nominalizador de nome de ação' 
$\{-r i\}$

(42) a. kmẽ-ri catar-NNA

'(o) catar'

b. wa-ri pedir-NNA

'(o) pedir'

c. kə-ri

pegar-NNA

'(o) pegar'

d. ta-ri apanhar-NNA

'(o) apanhar'

e. du-ri

levar-NNA

'(o) levar, carregar'

(43) a. hi-ri

deixar-NNA

'(o) deixar'

b. $\quad k a=w a z ə-r i$

$3=$ roçar-NNA

'(o) roçar (de algo)'

c. kazə-ri

bater-NNA

'(o) bater'

d. ka=hi-ri

$3=$ cozinhar-NNA

'(o) cozinhar (de algo)'

(44) a. ka-ri

beliscar-NNA

'(o) beliscar'

b. saiku-ri

subir-NNA

'(o) subir' 

c. sazə-ri
parar- NNA
'parar'
$\begin{array}{ll}\text { d. } & \text { ka=zazə-ri } \\ & \text { 3-salvaguardar-NNA }\end{array}$
'(o) salvaguardar; assegurar (de algo)'

$\{-r \tilde{i}\}$

(45) a. tsi-ri

espirrar-NNA

'(o) espirrar'

b. wĩ-rĩ

matar-NNA

'(o) matar'

c. mõ-rĩ

andar-NNA

'(o) andar'

d. $\tilde{1}$-zdam-rĩ

3-beijar- NNA

'(o) beijar' (beijar de algo/alguém)

(46) a. wa-rĩ

salgar-NNA

'(o) salgar'

b. kutõ-rĩ

acabar-NNA

'(o) acabar'

c. sõm-rĩ

dar- NNA

'(o) dar'

d. sap-rĩ

esconder- NNA

'(o) esconder'

e. $\quad$ t $\varepsilon$-rĩ

escorrer-NNA

'(o) escorrer' (da água) 
(47) a. smĩ-zakse-si-kutõ-rĩ PERT-saber-REFL-acabar- NNA

'(o) esquecer' (lit. “(o) acabar do saber de si/próprio referente a algo”)

b. sasõm-rĩ

guardar- NNA

'(o) guardar'

c. $\quad$ sa-ri $\tilde{\mathbf{i}}^{6}$

atrapar.com.fogo-NNA

'(o) atrapar com fogo (de uma caça)'

d. wam-rĩ

sacudir-NNA

'(o) sacudir'

e. pã-rĩ

matar-NNA

'(o) matar (matar algo em dois)'

$\{-r\}$

(48) a. wa-r

correr-NNA

'(o) correr'

b. kə-r

pegar- NNA

'(o) pegar'

c. du-r

carregar- NNA

'(o) carregar'

d. tsita-r

arrebentar- NNA

'(o) arrebentar'

e. kahi-r

cozinhar-NNA

'(o) cozinhar'

${ }^{6}$ A palavra $\{$ sarĩ $\}$ designa a técnica Xerente de atrapar, prender, aprisionar ou inibir um bicho no seu habitat (tatu ou cutia no buraco, por exemplo), utilizando fogo. 
(49) a. kahu-r engolir-NNA

'(o) engolir'

b. hu-r

copular-NNA

'(o) copular'

c. də-r

falecer-NNA

'(o) falecer'

d. sanãm-r

ler-NNA

'(o) ler'

(50) a. k-mã=nã-r

3-DAT=fazer-NNA

'(o) fazer (de algo)'

b. $\mathrm{ka}=\mathrm{nh} ə-\mathbf{r}$

$3=$ cortar - NNA

'(o) cortar (de algo)'

c. k-mã=kə-r

3-DAT=acertar-NNA

'(o) acertar (de algo)'

d. k-mã=tob-r

3-DAT=atravessar-NNA

'(o) atravessar (de/para algo/alguém)'

(51) a. wapa-r

ouvir-NNA

'(o) ouvir'

b. waza-r

misturar-NNA

'(o) misturar'

c. waptã-r

cair- NNA

'(o) cair'

d. hə-r

gritar-NNA

'(o) gritar' 
$\begin{array}{ll}\text { e. } & \text { wa-r } \\ & \text { correr-NNA } \\ & \text { (o) correr' }\end{array}$

(52) a. k-mã=nã-r

3-DAT =fazer-NNA

'(o) fazer (de algo)'

b. nmĩ =pa-r

PERT=esperar- NNA

'(o) esperar'

c. $\mathrm{k}-\mathrm{m} \tilde{e}=\mathrm{wa}-\mathbf{r}$

3 -Ass $=$ quebrar - NNA

'(o) quebrar, abrir (feijão/côco)

(53) a. kəi+kahu-r

água-tomar-NNA

(o) tomar (água)'

b. waza-r

misturar-NNA

'(o) misturar'

c. waiwẽ-r

balançar-NNA

'(o) balançar'

d. sa-r

morder-NNA

'(o) morder'

$\{-\varnothing\}$

(54) a. pke- $\varnothing$

bater-NNA

'(o) bater'

b. rmẽ- $\varnothing$

deixar-NNA

'(o) deixar'

Conforme demonstramos nos exemplos anteriores, a nominalização em Xerente é um processo de formação de nomes a partir de verbos. O morfema 
nominalizador de nomes de ação tem os seguintes alomorfes: $\{-r \tilde{1} \sim-r i \sim-r \sim$ $-\varnothing\}$.

Verbos em Xerente são nominalizados, portanto, pelo nominalizador de nome de ação. Esta nominalização é básica. As nominalizações que resultam em nome de ação podem ser a base para as demais nominalizações, quais sejam, a nominalização de agente e a nominalização de circunstância, tratadas, a seguir.

\section{Nominalizador de nomes de agente $\{-k w a\}$}

Nomes de agente são formados a partir da combinação de temas verbais combinados com o nominalizador de nome de ação e com o nominalizador $\{-\mathrm{kwa}\}$ :
a. da=pã-r-kwa
GEN.HUM=matar-NNA-NAG
'matador (de gente)'
b. sasa-r-kwa
caçador-NNA-NAG
'caçador'
d. da=pke- $\varnothing-\mathbf{k w a}$
GEN.HUM=bater-NNA-NAG
'batedor (de gente)'
e. $\quad$ rom $=$ kre- $\varnothing$-kwa
GEN.HUM-plantar-NNA-NAG
'plantador (de algo)'

(56) a. da=həi-kahu-r-kwa

GEN.HUM=pele-comer-NNA-NAG

'comedor de pele (de gente)'

b. si-re-kahu-r-kwa

ave-ATN-comer-NNG-NAG

'comedor de passarinho'

c. da=kahu-r-kwa

GEN.HUM-comer-NNA-NAG

'comedor de gente'

d. da=pa-kahu-r-kwa

GEN.HUM=figado-comer-NNA-NAG

'comedor de fígado (de gente)' 
e. $\quad$ da $=$ rmẽ- $\varnothing-\mathbf{k w a}$

GEN.HUM-deixar-NNA-NAG

'deixador (de gente)'

(57) a. da=rerke- Ø-kwa

GEN.HUM=derrubar-NNA-NAG

'derrubador (de gente)'

b. da=kə-r-kwa

GEN.HUM=pegar-NNA-NAG

'pegador'(de gente)'

c. da=hi-kẽ-Ø-kwa

GEN,HUM=perna-quebrar-NNA-NAG

'(o) quebrador de perna (de gente)'

d. da=painõ-kẽ-Ø-kwa

GEN.HUM=braço-quebrar-NNA-NAG

'(o) quebrador de braço (de gente)'

e. da=nõkrkre-ta-r-kwa

GEN.HUM=garganta-tirar-NNA-NAG

'(o) tirador de garganta (de gente)'

f. da=nõito-ta-r-kwa

GEN.HUM=língua-tirar-NNA-NAG

'(o) tirador de língua (de humano)'

Passemos ao terceiro tipo de nominalizador encontrado na língua Xerente, o nominalizador de nomes de circunstância.

\section{Nominalizador de nomes de circunstância $\{-z \varepsilon\}$}

Nomes de circunstância são formados em Xerente por meio da combinação de temas verbais nominalizados por meio do nominalizador de nome de ação, acrescidos do nominalizador $\{-\mathrm{z} \varepsilon\}$ :

(58) a. da=pã-r-ze

GEN.HUM $=$ matar-NNA-CIRC

'objeto de matar gente; lugar de morrer de gente'

b. $\mathrm{da}=$ nõkrkre- $\varnothing-\mathbf{z} \varepsilon$

GEN.HUM=garganta-NNA-CIRC

'dor de garganta de gente' 
c. $\quad$ da $=$ nmrã $-\varnothing-z \varepsilon$

GEN.HUM=sentar-NNA-CIRC

'lugar de assento (de gente)'

d. da=zam- $\varnothing-\mathbf{z} \boldsymbol{\varepsilon}$

GEN.HUM=em.pé-NNA-CIRC

'lugar de ficar em pé (objeto para ficar em pé de gente)'

e. da $=$ wra- $\varnothing-\mathbf{z} \varepsilon$

GEN.HUM=correr-NNA-CIRC

'lugar de correr (de gente)'

(59) a. da=nẽm- $\varnothing-\mathbf{z \varepsilon}$

GEN.HUM=andar-NNA-CIRC

'lugar de andar (de gente)'

b. da=siwaktu- $\varnothing-\mathbf{z} \varepsilon$

GEN.HUM=descansar-NNA-CIRC

'lugar de descanso (de gente)'

c. $\mathrm{da}=\mathrm{nt} \tilde{\mathrm{o}}-\varnothing-\mathbf{z \varepsilon}$

GEN.HUM=dormir-NNA-CIRC

'lugar de dormir (de gente)'

d. $\mathrm{da}=$ sihə-zu-m- $\varnothing-\mathbf{z} \varepsilon$

GEN.HUM=brincar-?-NNA-CIRC

'lugar de brincar (de gente)'

(60) a. da=nhə-r-ze

GEN.HUM=cortar-NNA-CIRC

'objeto ou local de cortar; faca'

b. $\mathrm{da}=$ nĩm-hrə- $\varnothing-\mathbf{z} \varepsilon$

GEN.HUM=PERT-tocar-NNA-CIRC

'lugar ou instrumento de tocar; teclado'

c. $\mathrm{da}=\mathrm{dk} \partial+\mathrm{nre \tilde { }}-\varnothing-\mathbf{z \varepsilon}$

GEN.HUM=morrer + nrẽ-NNA-CIRC

'lugar de sepultar, lugar de morte (cemitério)'

\section{Nominalizador de predicados existenciais}

A língua Xerente possui um morfema derivacional que deriva predicados existenciais e / ou estativos. Trata-se do sufixo $\{-d i\}$ e de suas variantes $-k i$ e -ti. Combinam-se com $\{-\mathrm{di}\}$ nomes e verbos nominalizados, como mostram os seguintes exemplos: 


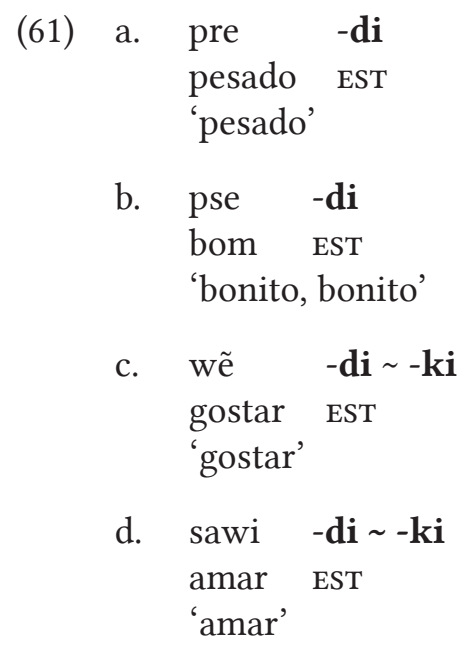

(62) a. sdakrı -di - -ki

sol EST

'(fazer) sol quente'

b. həze - $\mathbf{d i} \sim-\mathbf{k i}$

doente EST

'estar doente'

c. hrã wẽ $\quad-\mathbf{d i} \sim-\mathbf{k i}$

gritar bonito EST

'gritar bonito; gostar de gritar'

d. hə $-\mathbf{d i} \sim-\mathbf{k i}$

frio EST

'estar frio'

(63) a. mrmẽ zeĩ -di

falar DES EST

'gostar de falar'

b. wra tª -di

correr duro EST

'correr duro/muito'

\section{O morfema privativo}

Como descrito por Cotrim (2016), os alomorfes do morfema privativo do Xerente $\{-\mathrm{kõ}\} \sim\{$-tõ $\}$ contribuem com o significado de 'privado de, sem, destituído de algo'. Combinam-se com expressões nominais - nomes e verbos nominalizados. Alguns exemplos são: 
Exemplos com nomes

(64) a. sahi-kõ-di

cabelo-PRIV-EST

'careca' (lit.: "sem cabelo")

b. stikrui-kõ-di

ira-PRIV-EST

'tranquilo' (lit.: "sem raiva")

c. spokrep-tõ-di

orelha-PRIV-EST

‘surdo' (lit.: "sem ouvido")

d. smĩzawi-kõ-di

generosidade-PRIV-EST

'sem dó' (lit.: "sem genenerosidade")

(65) a. mrõĩ-kõ-di

cônjuge-PRIV-EST

‘solteiro' (lit.: "sem cônjuge")

b. krai-kõ-di

cria-PRIV-EST

'estéril' (lit.: "sem filho")

c. kwai-kõ-di

dente-PRIV-EST

'desdentado' (lit.: "sem dente, sem fio")

d. top+sui-kõ-di

olho+pelo-PRIV-EST

'sem cílios' (lit.: "sem pelo de olho")

e. brui-kõ-di

roça-PRIV-EST

'sem roça'

Exemplos com verbos nominalizados

(66) a. wra-Ø-kõ-di

correr-NML-PRIV-EST

'sem (o) correr' 
b. mrmẽ-Ø-kõ-di

falar-NML-PRIV-EST

'mudo' (lit.: "sem (o) falar")

c. kr-mõ-r-kõ-di

CONT-andar-NML-PRIV-EST

'sem (o) andar'

d. te-wapa-r+pse-kõ-di

3-ouvir-NML+bom-PRIV-EST

'sem (o) ouvir bem dele'

Após apresentamos os tipos de processos derivacionais encontrados na língua Xerente, passemos a algumas considerais finais sobre os mesmos.

\section{Algumas considerações finais}

O objetivo do presente trabalho foi o de descrever processos derivacionais encontrados no Xerente. Identificamos, até o momento, onze morfemas derivacionais Xerente. Os morfemas derivacionais aqui tratados são de natureza sufixal.

Os morfemas derivacionais do Xerente contribuem para a derivação de novos lexemas na língua. Há morfemas que são afixados a (a) temas nominais, verbais e adverbiais, b) temas nominais, (c) temas verbais, e (d) temas verbais nominalizados. Mostramos que os morfemas derivacionais são de diferentes naturezas, alguns formam novos lexemas da mesma classe da base, outros formam lexemas de classes distintas.

Salientamos que as palavras derivadas não obedecem a uma sistematização e obrigatoriedade para toda uma classe homogênea do léxico (Gleason 1955). De forma análoga, a derivação, ou melhor, os sufixos derivacionais em Xerente não atribuem necessariamente uma mesma semântica aos temas com os quais se combinam. Os morfemas derivacionais não constituem, assim, um quadro regular e preciso, tendo em vista que uma derivação pode ter como base um dado tema e não um tema congênere. Isso vai ao encontro do exposto por Aikhenvald (2015:50) quanto ao contraste entre flexão e derivação. Consoante Aikhenvald, as categorias flexionais são tipicamente regulares e previsíveis, em forma e em significado, ao passo que, categorias derivacionais, ao contrário, podem ser idiossincráticas. As derivações devem, portanto, ser listadas no léxicon e a história derivacional de cada palavra deve ser descrita separadamente.

\section{Referências}

Aikhenvald, Alexandra Y. 2015. The art of grammar: a practical guide. Nova York: Oxford University Press. 
Costa, Lucivaldo. 2013. Elementos para uma gramática da língua Xikrín. Tese de Doutorado em Linguística, Universidade de Brasília.

Cotrim, Rodrigo, G. P. M. 2016. Uma descrição da morfologia e de aspectos morfossintáticos da língua Akwẽ-Xerente (Jê Central). Tese de Doutorado em Linguística, Universidade de Brasília.

Gleason Jr., H. A. 1955. An introduction to descriptive linguistics. Nova York: Holt, Rinehart and Winston Inc.

Miranda, Maxwel G. 2014. Morfologia e morfossintaxe da língua krahô (família jê, tronco macro-jê). Tese de Doutorado em Linguística, Universidade de Brasília.

. 2010. Nominalizações na sintaxe da língua Krahô (Jê). Mestrado em linguística, Universidade de Brasília.

Rodrigues, A. D. Morfologia do verbo tupi. 1953. Letras. Curitiba 1: 121-152.

. Estrutura do Tupinambá. [1980] 2010. In: Ana Suelly Arruda Câmara Cabral e Aryon Dall'Igna Rodrigues (Orgs.). Línguas e Culturas Tupí 2. Brasília, DF: LALI/ UnB, p. 167-203.

Rodrigues, A. D.; Cabral, A. S. A. C. Tupían. In: Lyle Campbell; Verónica Grondona (Org.). The Indigenous Languages of South America. Berlin/ Boston: Mouton de Gruyter, 2012, v. 2, p. 495-574.

Recebido em março de 2017.

Aceito em maio de 2017. 